\title{
Bidirectional flow measurement based on the differential pressure method for surge analysis on a small centrifugal compressor
}

\author{
Moritz Werner', Roland Baar', Peter Haluska ${ }^{2}$ and Ivo Sandor ${ }^{2}$
}

\begin{abstract}
To obtain a high temporal resolution of mass flow data, a flowmeter based on the differential pressure method has been developed. It is capable of detecting negative flow for investigations of dynamic effects in small centrifugal compressors used for turbocharging automotive internal combustion engines. Experiments were performed at a hot gas test bench focusing on the surge characteristics at different turbocharger speeds and the influence of volume modifications downstream of the compressor. Instantaneous operating points could be traced in the compressor map including the typical orbits at deep surge resulting from the cyclic character of the phenomenon.
\end{abstract}

\section{Keywords}

Centrifugal compressor, flow measurement, flowmeter, Venturi effect, surge

Date received: 6 April 2016; accepted: 28 July 2016

\section{Introduction}

Surge is an unstable turbocharger compressor performance occurring at low flow rates and high pressure ratios. If the pressure gradient in the compressor is too high, flow separation and stalling of fluid within the impeller or diffusor can result in temporarily reversed flow. This causes a cyclic discharging and charging of the compressor outlet volume and leads to large oscillations in pressure and mass flow. Such an unstable operating mode must be avoided since it can severely damage the turbocharger and limit the functionality of the combustion engine attached to the compression system.

For an investigation of the cyclic phenomenon, the instantaneous operating points of the compressor during surge shall be traced. The surge phenomenon with respect to the geometry of the ducting system downstream of the compressor has first been analyzed and modeled by Greitzer, ${ }^{1}$ followed by numerous researches e.g. Galindo et al. ${ }^{2}$ and Kabalyk et al. ${ }^{3}$

Testing facilities for turbochargers of automotive engines are commonly designed to record steady-state performance maps. At these operating points, typically only one-directional, stable flow occurs. Hence, the common data acquisition rates of turbocharger test benches are in the range of $10-100 \mathrm{~Hz}^{4}{ }^{4}$ The oscillations during surge are of frequencies between 5 and $30 \mathrm{~Hz}^{5}$ An analysis of this phenomenon therefore demands higher time resolution of the data at rates $\geqslant 1 \mathrm{kHz}$. In order to achieve this, the sampling rate of the data acquisition hardware has to be increased and sensors have to be used with a suitable short latency and settling time.

Operating points of compressors are typically characterized by compressor speed, pressure ratio and flow rate. Since the speed measurement is generally based on counting the blades of the compressor wheel passing the sensor position, it can be easily adapted. The pressure can also be measured at suitable rates with well-proven techniques such as piezoresistive or piezoelectric sensors. However, the measurement of flow rate in this setting presents several challenges for commercially available solutions.

For the analysis of transient effects, it is necessary to minimize damping effects caused by volumes and piping. A sensor location very close to the inlet or outlet of the compressor is mandatory with only a small flow-calming section for the sensor itself. However, this sensor position calls for a measuring

\footnotetext{
'Institut für Land- und Seeverkehr, Fachgebiet

Verbrennungskraftmaschinen, Technische Universität Berlin, Berlin, Germany

${ }^{2}$ Continental Automotive $\mathrm{GmbH}$, Regensburg, Germany
}

\section{Corresponding author:}

Moritz Werner, Technische Universität Berlin, Institut für Land- und Seeverkehr, Fachgebiet Verbrennungskraftmaschinen, Carnotstraße I A, Berlin 10587, Germany.

Email: moritz.werner@tu-berlin.de 
system with a low impact of swirls or unbalanced flow profiles. Flow straighteners would also add to the damping volumes or their geometry might reduce the effect to be examined.

One of the fundamental requirements for a precise surge analysis is the reliable detection and measurement of negative flow. Systems that offer this feature, such as ultrasonic sensors or particle image velocimetry, require considerable investments. Hotwire probes are commonly used for this measuring task, e.g. by Grigoriadis. ${ }^{4}$ However, they do not contain a determination of the flow direction and are easily affected by asymmetric flow profiles. In addition they are rather fragile. Possible occurrence of oil droplets or dust particles should be considered though which must not impair the results or even damage the sensor.

To meet these objectives, a differential pressure flowmeter was designed to be installed directly at the compressor outlet, as shown in Figure 1. This position allows the indication of flow direction with a method based on pressure trends, which is explained further below in the text. Measurement techniques for pulsating flow with the differential pressure principle are proposed by Gajan et al. $^{6}$ and Dobelhoff-Dier et al. $^{7}$ using orifice plates as well as by Beaulieu ${ }^{8}$ using a Venturi tube for a liquid flow application. Venturi tube measurements of pulsating, unidirectional gas flow are described by Laurantzon ${ }^{9}$ and Reuter. ${ }^{10}$ In addition to the industry standards ${ }^{11,12}$ for the design and installation of such an instrument, Mottram's ${ }^{13}$ and Reader-Harris ${ }^{14}$ work were used as the basis for the development.

\section{Flowmeter design}

The flowmeter presented here uses the Venturi effect at a defined constriction inside the measuring device, which is built into a fluid leading conduit. Due to the reduction of cross section, the flow is accelerated at this throat and a static pressure drop from the duct of original flow area can be determined. This pressure difference characterizes the flow velocity and therefore the mass flow.

The flowmeter presented here has a symmetrical geometry with cylindrical sections at the inlet, throat and outlet with conical sections in between (Figure 2). It differs from typical Venturi tubes defined by the industry standard. ${ }^{12}$ One parameter in accordance with the norm and inside the proposed range is the diameter ratio of $\beta=D_{2} / D_{1}=0.74$, which results in a significant rise of velocity in the center part. This leads to high differential pressures that are easier to detect, especially if the flow is subject to pressure fluctuations. However, the geometry was designed so that Mach numbers in the throat do not exceed 0.3 at steady-state operating points. In addition, rather high $\beta$-values minimize the influence of the flowmeter on the flow and the phenomenon to be examined.

Since the flowmeter itself holds a certain volume of fluid, draining effects occurring during the change of flow direction have to be minimized. During the designing process, different length/diameter ratios $L / D_{1}$ for the flowmeter have been tested at the test bench. The evaluation resulted in a minimized short length/diameter ratio of $L / D_{1}=1.79$ with a high opening angle of $\delta=30^{\circ}$ on both sides.

The symmetrical design is the basis for the analysis of reverse flow. Therefore, the flowmeter is equipped with a sensor set at the main flow inlet (A) and at outlet (B). The measurement planes are located halfway along each of the cylindrical sections. Prior to the measurements, computational fluid dynamics analysis was used to investigate the locations. For the simulation, the flowmeter was connected to a fully modeled compressor side of the turbocharger. Further information on the compressor simulation are described by Schäfer. ${ }^{15}$ The results calculated for one line of constant compressor speed showed consistent values

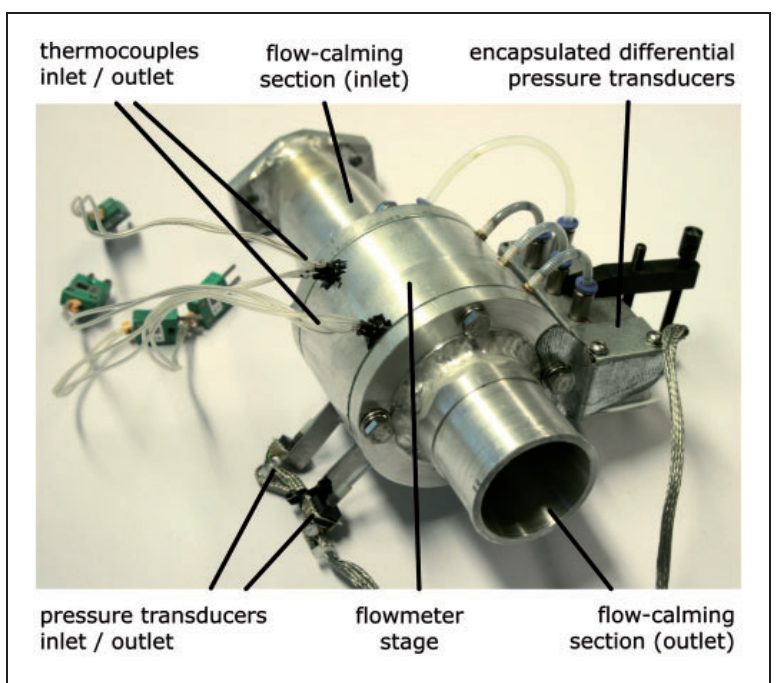

Figure I. Differential pressure flowmeter.

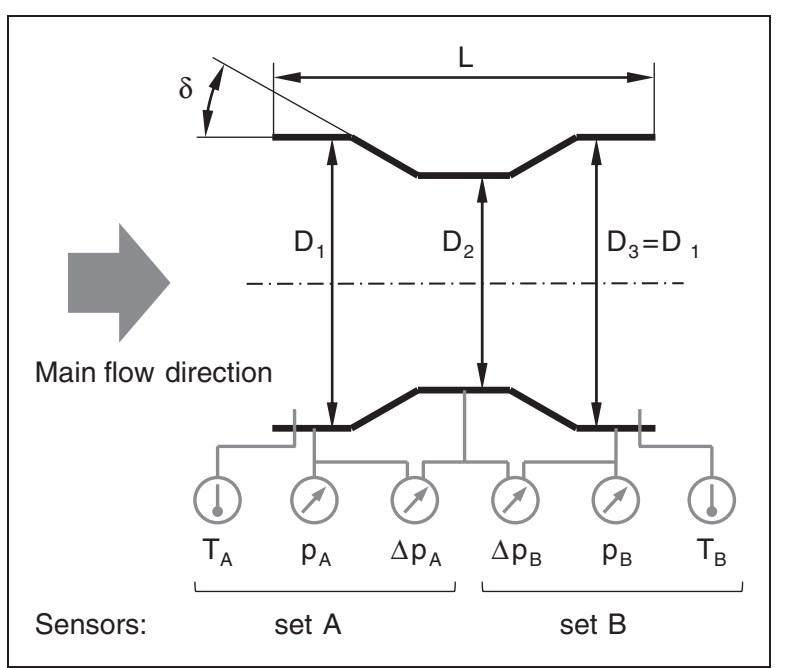

Figure 2. Schematic representation of the flowmeter. 
of the flow rate coefficient $c_{D}$ of the flowmeter, although swirl effects increase with higher mass flows and are dominant at compressor operating points close to choke. Swirl flow should therefore only have a negligible influence on the measurement results.

Differential pressure sensors are installed between the large and the narrowed flow section, $\Delta p_{A}$ and $\Delta p_{B}$. For the large cross-sectional areas, pressure and temperature are also measured to determine the fluid density. For a temporal resolution of $1 \mathrm{kHz}$, pressure sensors with a response time of $0.5 \mathrm{~ms}$ and limited internal volume are installed. They are connected with the flow through small bore holes in the wall of the flowmeter. The temperature is measured by open thermocouples in the near-wall region.

The simple geometry and setup of the flowmeter makes it insensitive to dust particles and oil droplets. Nevertheless, a single-phase flow is generally assumed.

\section{Determining the resulting mass flow}

Applying Bernoulli's law, an absolute value for the mass flow through the flowmeter can be calculated with knowledge of the differential pressure towards the throat, the fluid density and the diameters of the cross-sectional areas. ${ }^{11}$ The approach derives from the simple assumption of steady-state flow but will be used in this application.

$$
\begin{aligned}
\dot{m} & =c_{D} \cdot \varepsilon \cdot \dot{m}_{i d} \\
& =c_{D} \cdot \varepsilon \cdot \frac{\frac{\pi}{4} \cdot D_{2}^{2}}{\sqrt{1-\left(\frac{D_{2}}{D_{1}}\right)^{4}}} \cdot \sqrt{2 \cdot \frac{p}{R \cdot T} \cdot \Delta p}
\end{aligned}
$$

Since the flow conditions cannot be assumed as frictionless and incompressible, the flow rate coefficient $c_{D}$ and the expansion number $\varepsilon$ have to be taken into account. The two factors are summarized to a joint correction factor $\mu$, which will be determined by a calibration measurement.

Both the physical positive flow and the negative flow result in a positive differential pressure measured with set A and set B sensors. Only absolute values for the mass flows $\dot{m}_{A}$ and $\dot{m}_{B}$ are calculated. However, it will be shown that the calculated trends differ from each other and, therefore, only the first set in the instantaneous flow direction will be considered. The values of the other set can be influenced by flow separation due to the large opening angles of the geometry.

The raw data offer no direct values to identify positive or negative flow. Hence, data processing is needed to set indicators for the instantaneous flow direction. The resulting mass flow is then calculated with values of $\dot{m}_{A}$ for the positive flow direction and $-\dot{m}_{B}$ for the negative flow direction. To ensure continuous values, $\dot{m}_{A}$ and $-\dot{m}_{B}$ are crossfaded over a short time range $t_{X F}$ at the change of direction. This is done by

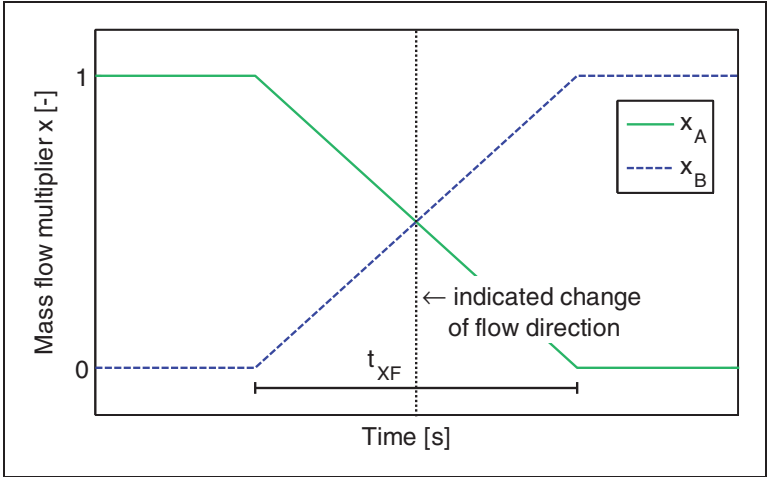

Figure 3. Mass flow multipliers for crossfade at indicated directional change towards negative flow $\left(\dot{m}_{A}\right.$ to $\left.-\dot{m}_{B}\right)$.

additional multipliers $x_{A}$ and $x_{B}$ with values ranging between 0 and 1 and a ramp function during the fading event

$$
\dot{m}_{i d}=x_{A} \cdot \dot{m}_{A}-x_{B} \cdot \dot{m}_{B}, \quad \text { with } x_{B}=1-x_{A}
$$

Figure 3 shows an example of the multipliers for a change of positive towards negative flow. The crossfade duration $t_{X F}$ is adjusted to the timescale of the surge cycle.

Two methods are considered to determine the indicators of direction: The analysis of the pressure gradient between $\mathrm{A}$ and $\mathrm{B}$ and an investigation of pressure value trends. A third approach, the determination of time lag (positive or negative) between two similar signals of sets A and B was not examined here because of signal noise and the higher sampling rates required.

\section{Experimental setup}

The experiments were carried out on a small centrifugal compressor of a turbocharger for an automotive gasoline engine with a displacement of 11 . The hot gas test bench and its setup are documented by Pucher. ${ }^{16}$ The compressor side was operated in an open-loop mode. Ambient air of the test cabin enters the compressor through the standard measuring setup for mass flow, pressure and temperature as shown in Figure 4. Downstream of the turbocharger compressor is the differential pressure flowmeter, the standard measuring equipment of the discharge side, a flow volume, and a throttle valve to build up back pressure.

For high temporal resolution pressure measurement and determination of compressor pressure ratio, additional Kulite pressure probes are installed in the compressor housing at inlet and outlet.

The test program focusses on operating conditions during compressor surge as well as steady-state operating points for calibration of the flowmeter. Turbocharger speed and flow volume were varied. The total volume of the compressor discharge side 
for a large flow volume setup is 13.21 and 1.31 for the small flow volume setup.

All pressure measurements of the flowmeter have been digitally low-pass filtered with zero-phase shift.

\section{Results}

\section{Calibration measurement}

The flowmeter was calibrated at steady-state operating points considering the entire range of the compressor map and both flow volumes. With the use of a proven standard measurement for mass flow $\dot{m}_{\text {ref }}$ at the test bench, the correction factor $\mu$ was determined as

$$
\mu=c_{D} \cdot \varepsilon=\frac{\dot{m}_{r e f}}{\dot{m}_{i d}}
$$

For a standardized Venturi tube of a defined geometry and a medium with an isentropic exponent assumed as constant, $\mu$ is primarily dependent on the pressure ratio between the small and large cross- sectional area sections. ${ }^{4}$ With higher flow rates or pressure differences, the influence of the compressibility of the gas increases, which leads to a decrease of $\mu$.

Following this approach, $\mu$ is examined with respect to differential pressures $\Delta p$. The data in Figure 5 show the same trend of a decreasing correction factor. The correction factor scatters over a wider range for normalized $\Delta p<0.25$. This data was collected close to the surge line where the performance of the compressor varies with speed and flow volume since it is highly influenced by the inherent unsteady nature of the flow field within the compressor. For further calculations, the correction factor is linearized as shown in Figure 5. Due to the symmetric design of the flowmeter, the trend of $\mu$ is assumed to be equal for both flow directions. The uncertainty of $\mu$ has been determined to be $5.6 \%$ for $\Delta p<0.25$ and $3.7 \%$ for $\Delta p \geqslant 0.25$.

Different turbochargers were not tested with the flowmeter, but it is expected that recalibration will be mandatory for each test object. The flowmeter can therefore only augment the standard test bench measuring equipment.

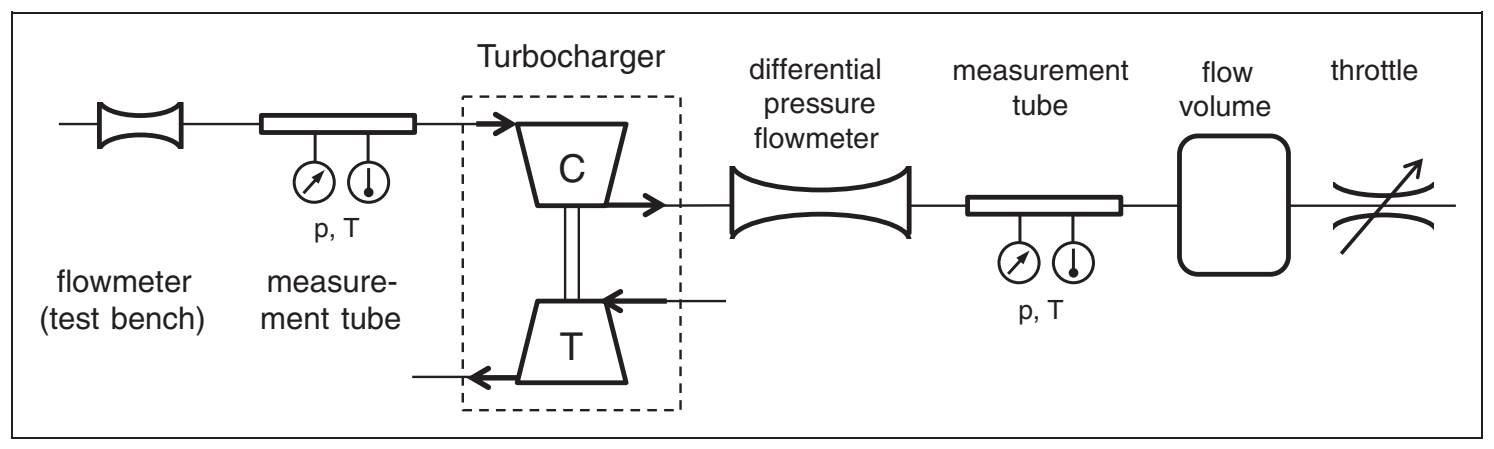

Figure 4. Turbocharger test bench, compressor air path.

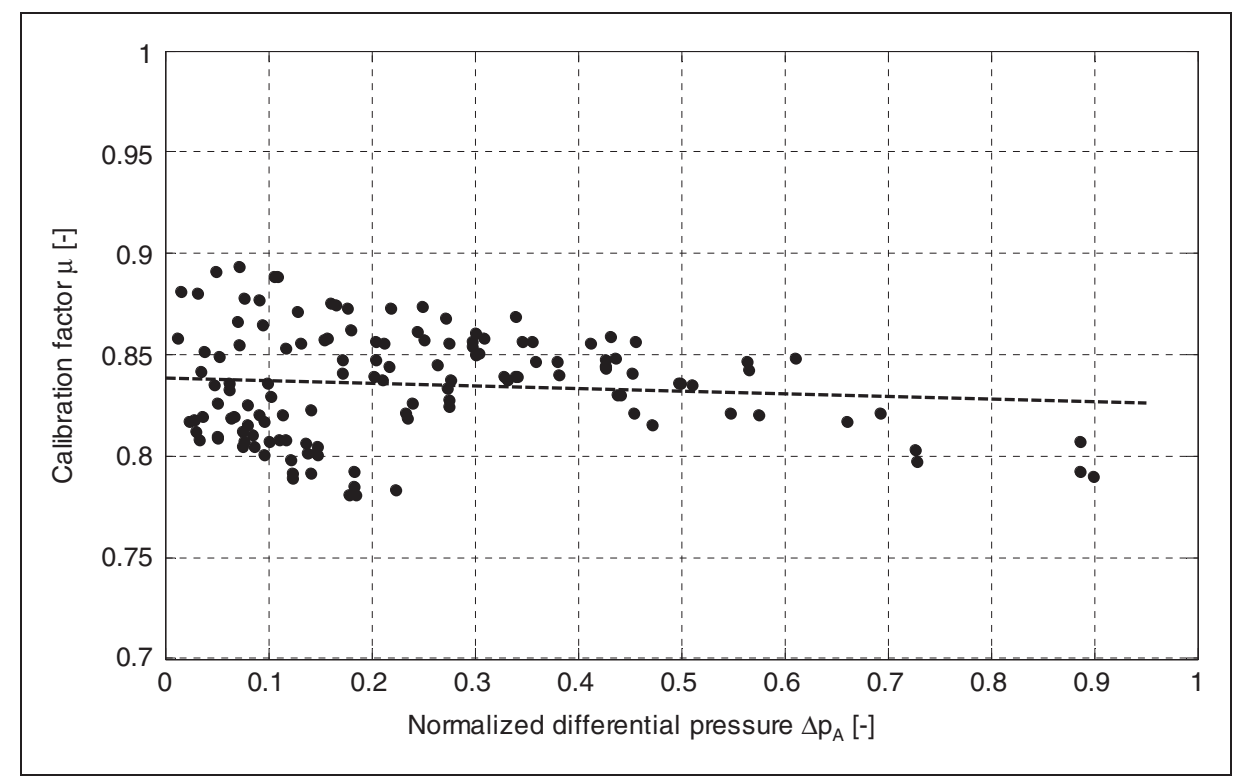

Figure 5. Calibration factor depending on the differential pressure. 


\section{Reverse flow indication}

Pressure gradient of inlet and outlet conditions. Only simple data processing is needed for the assumption of flow orientation following the instantaneous pressure gradient. This kind of approach can be found in simple 0-dimensional process simulations neglecting physical distances between two sensed positions. Regarding the setting investigated here, the distance, volume and therefore the inertial effects between the pressure sensors $p_{A}$ and $p_{B}$ are not considered, which may lead to imprecise indicators of direction.

Figure 6(a) shows the normalized raw, unfiltered data of inlet and outlet pressure $p_{A}$ and $p_{B}$ with a small flow volume setup at surge onset. The calculated normalized pressure difference is positive before and during surge, fluctuating around 0.03 (Figure 6(b)). This indicates no change in flow direction except for the two events marked with circles, where the gradient turns negative for an instant. Reducing the pressure difference to examine the possible effect of a constant sensor deviation due to poor calibration leads to a higher number of indicators. For a reduction of e.g. 0.02 the distribution of the indicators (marked with crosses) is spread randomly during the pulsating flow within all parts of the single surge events. They even occur under stable conditions, contradicting the actual physical behavior. Filtering neither leads to constant positive flow at the beginning of the measurement nor does it show a regular pattern during surge. Due to the impact of inertial effects inside the flowmeter this method does not offer a suitable solution.
Analysis of pressure trends. During surge, periodic oscillations in pressures $p_{A}$ and $p_{B}$ are dominant. After a short windup, the pressure decreases rapidly at surge onset. Applying the equation of state this significant pressure drop in the flowmeter and duct downstream the compressor can only be the result of mass leaving this subsystem. Since the maximum flow through the throttle valve of the test bench is already reached, the pressure loss must be due to reverse flow through the compressor. Determination of the local pressure maxima and minima leads to indicators for the change of flow direction. For the same data as presented above, the beginning of reverse flow is marked with circles in Figure 7, and the change to a positive flow direction is shown with squares.

During periods of the surge cycle where the pressure is dropping, the negative mass flow data of sensor set $\mathrm{B}$ is taken to calculate the resulting flow, otherwise positive values of the inlet are taken into account. At flow reversal, both the two data sets are crossfaded to avoid discontinuities.

\section{Mass flow analysis at compressor surge}

With a large flow volume integrated in the air path the surge frequency is $5 \mathrm{~Hz}$. For a turbocharger operating mode with medium speed, the resulting mass flow at deep surge is illustrated in Figure 8. The values cover the flow determined with sensor A for positive flow and B for negative flow. The data reflect the periodic behavior of the surge cycles. For each cycle, the flow in the positive direction is similar in amplitude and shape whereas the trends for the negative flow direction vary widely. The values not considered are of smaller

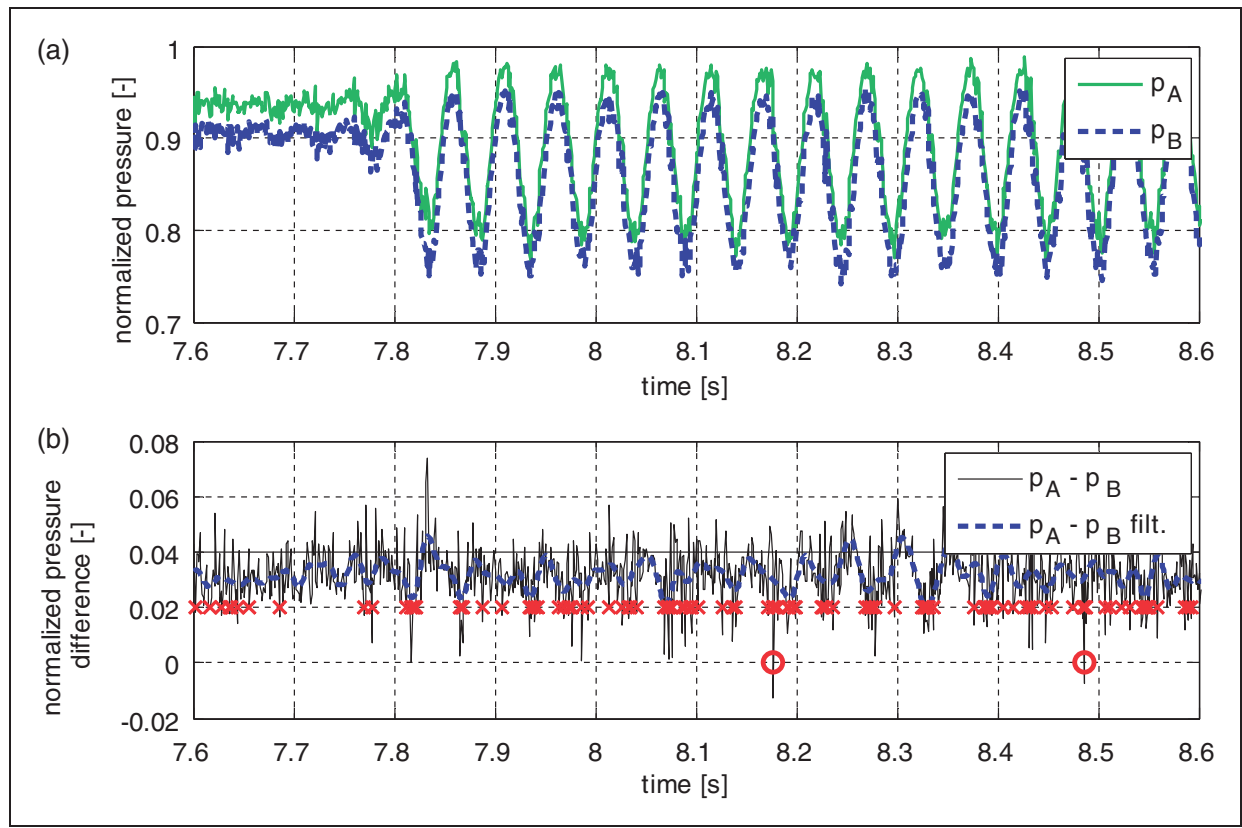

Figure 6. (a) Raw data of pressure fluctuations at the inlet and outlet of the flowmeter during surge onset and surge. (b) Calculated pressure difference of raw and filtered data with indicators for reverse flow. 


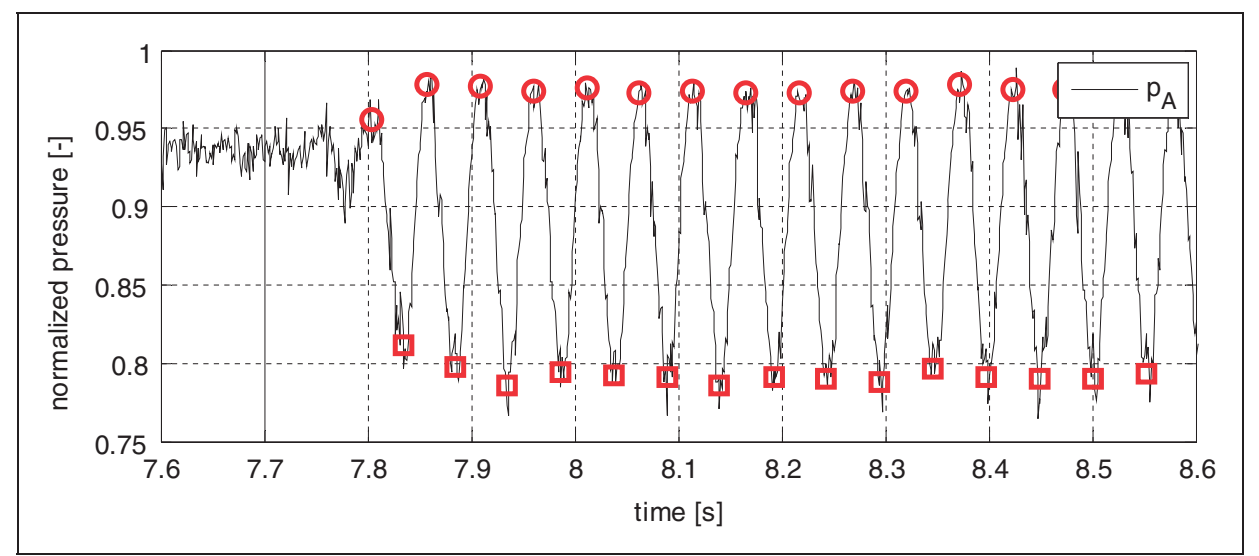

Figure 7. Raw data of pressure fluctuations at the inlet of the flowmeter during surge onset and surge with directional indicators based on pressure trend analysis.

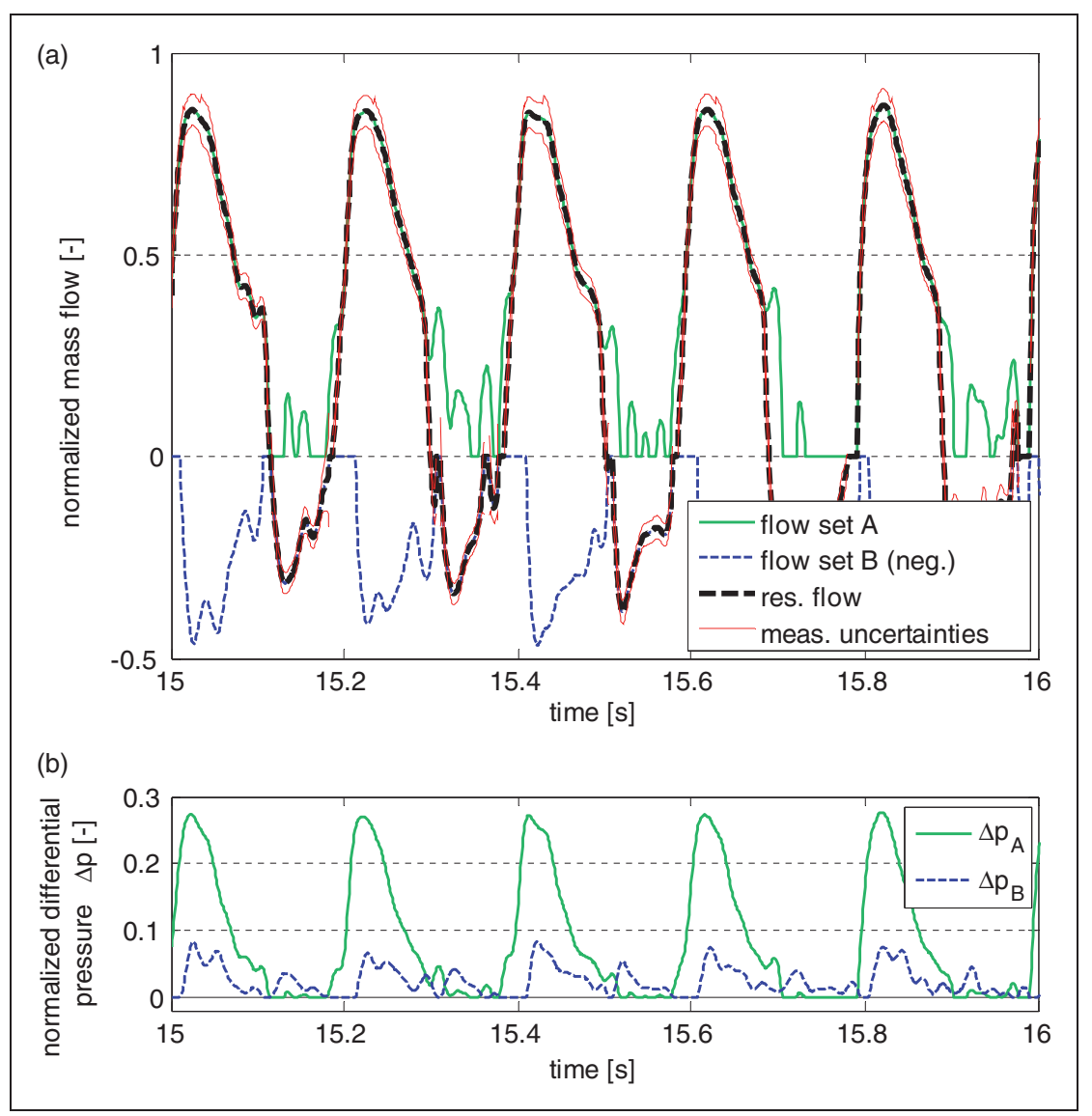

Figure 8. (a) Mass flow and (b) measured differential pressures $\Delta p_{A}$ and $\Delta p_{B}$ during deep surge with a setup of a large flow volume.

amplitude but show similar characteristics, in particular the mass flow values calculated with set $\mathrm{B}$.

In addition, the measurement uncertainty of the resulting mass flow is graphed. It has been calculated according to the law of propagation of errors to obtain a combined uncertainty for the measuring device from the measurement uncertainties of each single internal sensor. A deeper analysis has shown that the uncertainty of the correction factor $\mu$ has the most significant impact, followed by the measurement uncertainties of pressure and temperature. For all deep surge measurements shown in the sections below, a cycle averaged combined uncertainty has been calculated. The mean value of these uncertainties with reference to the overall maximal normalized mass flow of 1.79 is $2.4 \%$.

Figure 9 shows deep surge of the same speed with a small flow volume. An increased surge frequency of $19 \mathrm{~Hz}$ can be found due to the faster filling and discharging processes in comparison to the large flow volume. The trends appear to show a more uniform pulsing character at positive flow. 


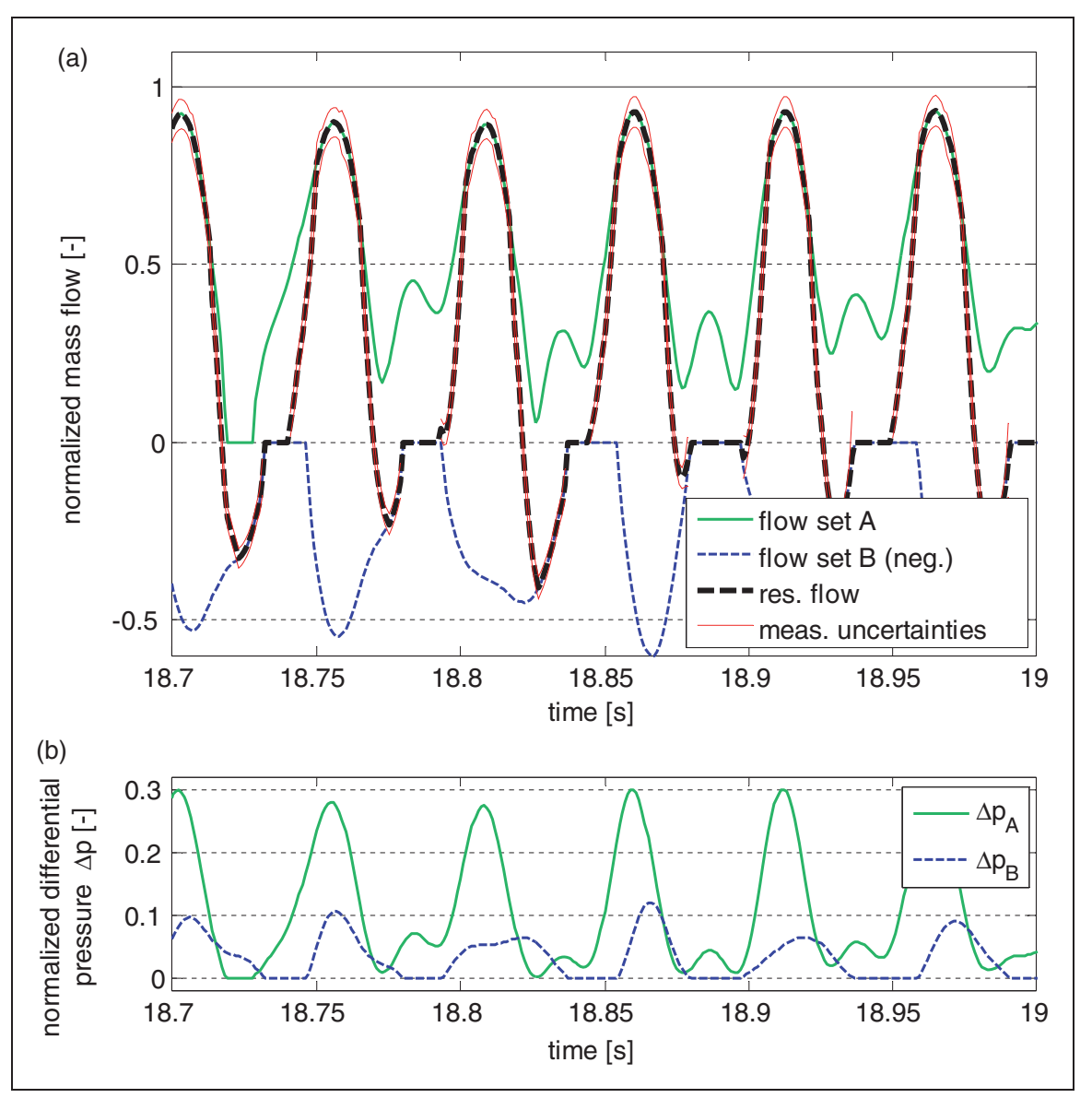

Figure 9. (a) Mass flow and (b) measured differential pressures $\Delta p_{A}$ and $\Delta p_{B}$ during deep surge with a setup of a small flow volume.

At some instances the resulting mass flow has a value of zero over a couple of samples, mostly occurring in data measured with small flow volume. But for a dynamic process this does not represent physical behavior. The effect occurs even more frequently and for longer periods of time in the individual sets of mass flow data not considered for the resulting flow.

These zero values have their origin in the differential pressure shown in Figures 8(b) and 9(b). Some data trends suggest the change to negative $\Delta p$, but this results in complex mass flow values that do not find a physical interpretation. The origin of the zero data could not be clarified satisfactorily. The most likely reasons are the pressure sensor settling time, effects in the connecting tubes of the sensor as well as distortions, pressure oscillations, draining effects, or other phenomena in the flow that cause negative values of $\Delta p$, which were not recorded with the unidirectional sensors.

\section{Transient compressor surge operating points in the compressor map}

The classical compressor map is used to trace the transient operating points of the surge cycle. In addition to the transient data, the corresponding speed line of steady-state compressor operating points is shown as a reference. The instantaneous total compressor pressure ratio is recorded through the Kulite pressure probes.
Eight surge cycles of the same speed as in Figures 8 and 9 are shown in Figure 10 for large flow volume and in Figure 11 for small flow volume. Each surge cycle starts near the end of the steady-state speed line at the point where the impeller cannot sustain the low flow rate at high pressure ratio. The mass flow drops rapidly from positive to negative values resulting in the reverse flow from the flow volume back through the compressor. The reverse flow causes the pressure ratio to drop. As soon as the compressor starts conveying air again, a rapid increase of flow in the positive direction is recognized along with an increase of pressure ratio. The operating point approaches the steady-state speed line and follows close to it while the flow volume fills again. This results in rising pressure ratios and decreasing flow. If the test bench throttle position has not changed, the mass flow breaks in again and the cycle repeats.

For each flow volume setup, the data of positive flow are in good accordance with the single cycles. Deviations are largest at the change of flow direction in particular at the onset of positive flow. During negative flow the paths show larger variations. For the large volume, the data scatter most while discharging from 0.87 to 0.8 of normalized total compressor pressure ratio. Below 0.8 the data points cluster and are in good agreement with a quadratic function. This complies with a turbine characteristic 


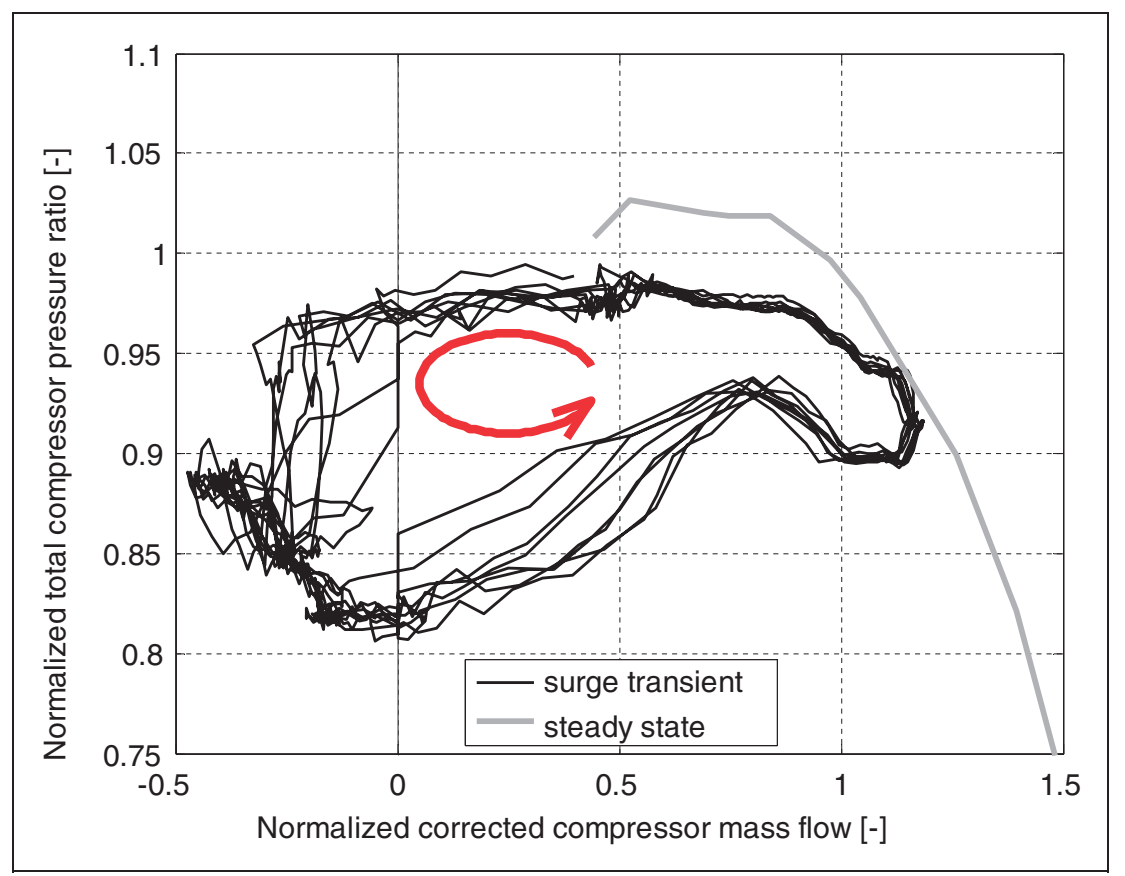

Figure 10. Transient compressor operating points during surge, large flow volume.

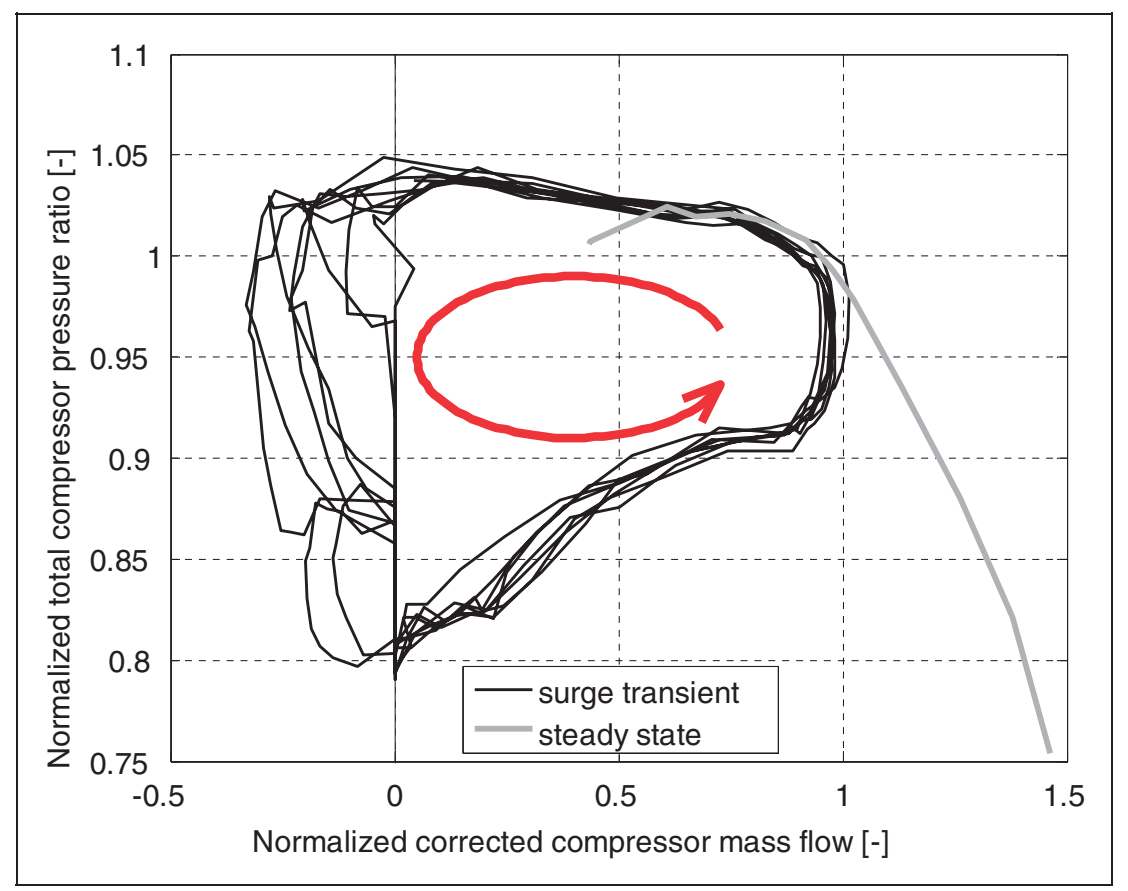

Figure II. Transient compressor operating points during surge, small flow volume.

of the compressor during the discharging process. A trend like this cannot be found in the data recorded with the small flow volume. There the pressure ratio drops almost linear with the decreasing flow rate.

\section{Analysis of averaged surge cycles}

For further analysis of the surge cycle paths, averaged cycles were calculated. To ensure uniform data, each of the single surge cycles of one measurement was interpolated to a constant vector length of 100 values. The single interpolated vectors were not filled with data at points of zero resulting mass flow. As a result, these points are not considered in the averaging process. An averaged cycle was calculated with data available of 20 to 25 previously interpolated surge cycles.

A comparative representation of the two flow volumes with the same turbocharger speed as presented above is given in Figure 12. The data orbit of the 


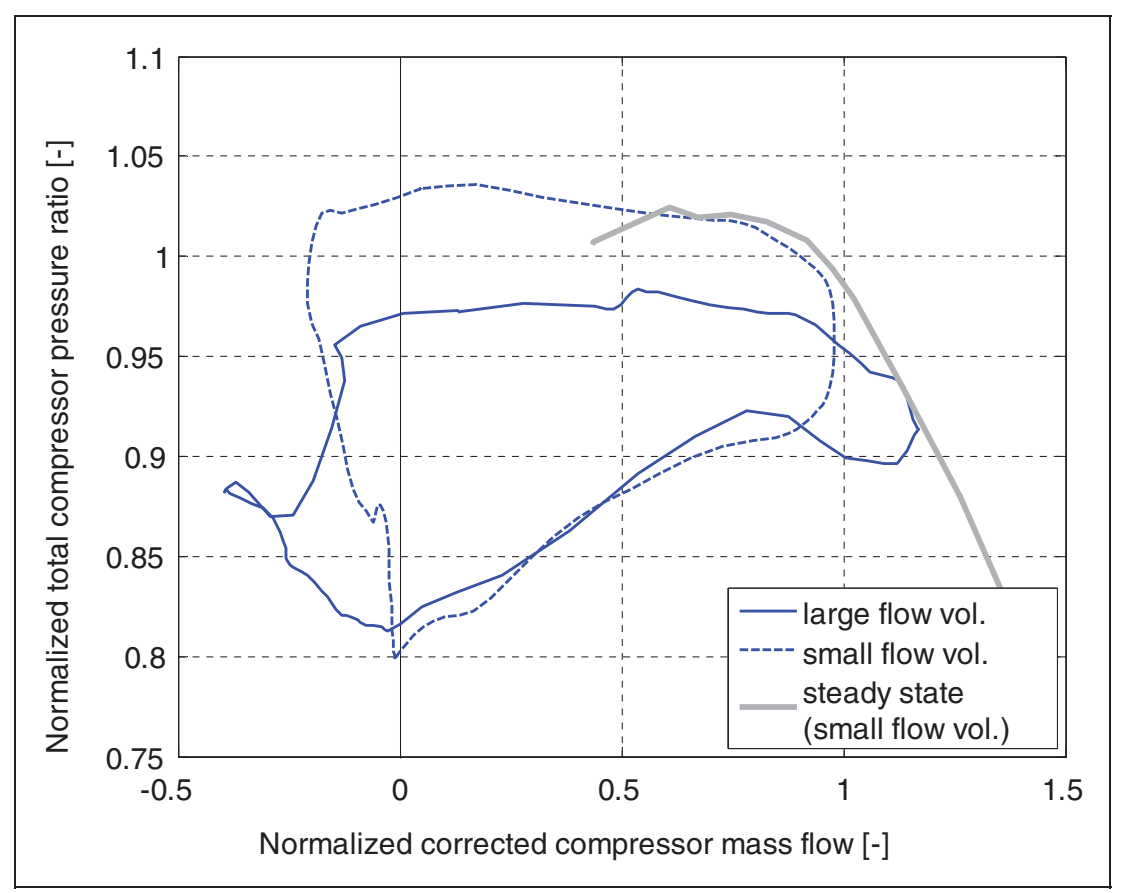

Figure 12. Averaged surge cycle of small and large flow volume setup.

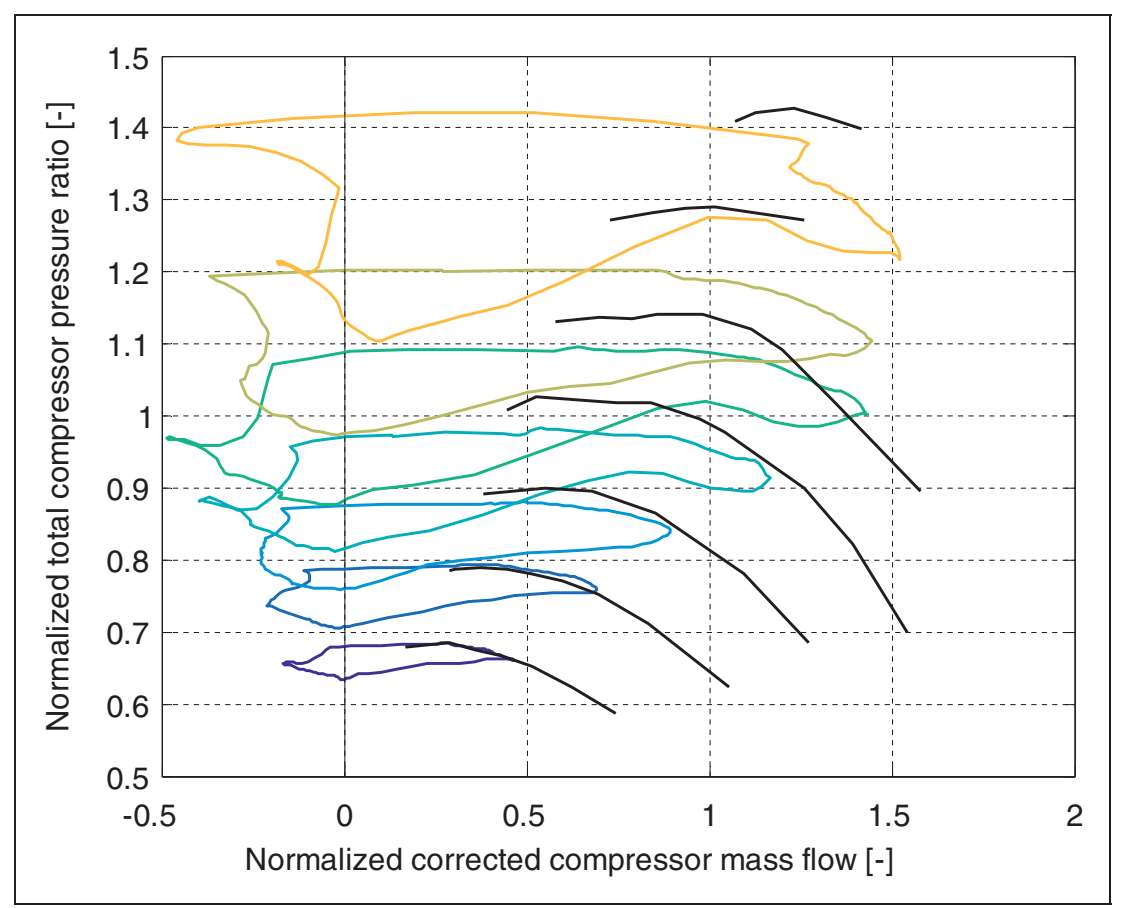

Figure 13. Averaged surge cycles for different compressor speeds and large flow volume setup.

small volume has a higher oscillation in pressure ratio and follows the characteristics at the top of the steady-state speed line. As the time resolved data show, the pressure drop during negative flow has a linear trend. In contrast, the large volume shows higher mass flow amplitudes but does not follow the speed line during mass flow decrease. This is not an effect of the mass flow determined but the pressure ratios measured since generally lower values prevail with this flow volume setup.

The averaged surge cycles for increasing compressor speeds (from lower at bottom to higher at top) are shown in Figure 13 (large flow volume) and Figure 14 (small flow volume). Steady-state operating points of high pressure ratio and flow rate have not been recorded in this measurement. The shapes of the 


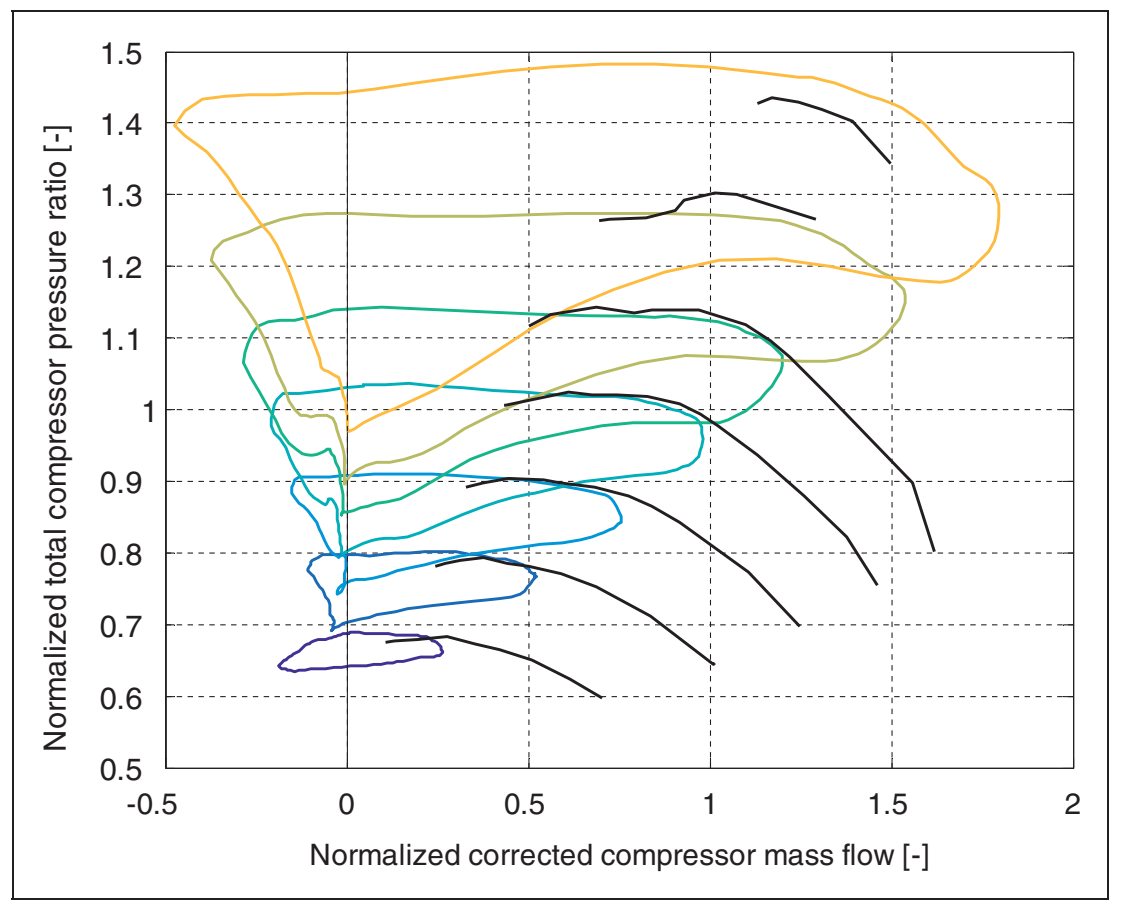

Figure 14. Averaged surge cycles for different compressor speeds and small flow volume setup.

averaged cycles shown in Figure 13 are in good agreement for low and medium speeds. The recorded cycle of second highest speed shows lower pressure ratio values than expected and the highest speed cycle differs from the other cycles in shape.

All surge orbits of small flow volume setup (Figure 14) are similar, except for the lowest speed line. Each cycle follows close to the relevant steadystate values with the exception of the orbit of highest speed, which exceeds the steady-state data points.

\section{Summary}

A flowmeter of high temporal resolution has been developed for the investigation of dynamics in small centrifugal compressors such as compressor surge. The measuring principle is based on the Venturi effect, which results from a section with reduced cross-sectional area inside the flowmeter. The symmetrical layout and configuration of the internal sensors allow a bidirectional flow measurement. The flowmeter has a compact design without a long flow-calming section. This opens up the opportunity to use it in other pulsating flow applications besides the investigations presented here, such as on-engine measurements where packaging constraints have to be considered.

Indicators have been examined for determining the flow direction. Compressor surge investigations were carried out for different turbocharger speeds and flow volumes downstream the compressor outlet. The time resolved mass flow characteristics were analyzed with respect to time and further processed to trace the instantaneous operating point in the compressor map. The data reflect the oscillating character of the surge phenomenon. For positive flow each of the single surge cycles are in good agreement; data of negative flows show larger deviations from cycle to cycle. A comparison of averaged surge cycles shows differences in the cycle shape with higher mass flow amplitudes for the large flow volume and higher pressure ratio amplitudes for the small flow volume. Variations in speed do not result in different characteristics but lead to scaled surge orbits for most operating modes.

\section{Declaration of Conflicting Interests}

The author(s) declared no potential conflicts of interest with respect to the research, authorship, and/or publication of this article.

\section{Funding}

The author(s) received no financial support for the research, authorship, and/or publication of this article.

\section{References}

1. Greitzer EM. Surge and rotating stall in axial flow compressors. J Eng Power 1976; 98: 190-217.

2. Galindo J, Serrano JR, Climent H, et al. Experiments and modelling of surge in small centrifugal compressor for automotive engines. Exp Therm Fluid Sci 2008; 32: $818-826$.

3. Kabalyk K, Liskiewicz G, Horodko L, et al. Use of pressure spectral maps for analysis of influence of the plenum volume on the surge in centrifugal blower. In: Proceedings of ASME Turbo Expo 2014, Düsseldorf, Germany, 16-20 June 2014, paper no. GT2014-26931. 
4. Grigoriadis P. Experimentelle Erfassung und Simulation instationärer Verdichterphänomene bei Turboladern von Fahrzeugmotoren. $\mathrm{PhD}$ Thesis, Technische Universität Berlin, Germany, 2008.

5. Pucher $\mathrm{H}$ and Zinner K. Aufladung von Verbrennungsmotoren. 4th ed. Berlin: Springer Vieweg, 2012.

6. Gajan P, Mottram RC, Hebrard P, et al. The influence of pulsating flows on orifice plate flowmeters. Flow Meas Instrum 1992; 3: 118-129.

7. Doblhoff-Dier K, Kudlaty K, Wiesinger M, et al. Time resolved measurement of pulsating flow using orifices. Flow Meas Instrum 2011; 22: 97-103.

8. Beaulieu A, Foucault E, Braud P, et al. A flowmeter for unsteady liquid flow measurements. Flow Meas Instrum 2011; 22: 131-137.

9. Laurantzon F. Flow measuring techniques in steady and pulsating compressible flows. Technical Report, KTH Mechanics, Stockholm, Sweden, 2010.

10. Reuter S. Erweiterung des Turbinenkennfelds von PkwAbgasturboladern durch Impulsbeaufschlagung. $\mathrm{PhD}$ Thesis, Technische Universität Dresden, Germany, 2010.

11. ISO 5167-1:2003. Measurement of fluid flow by means of pressure differential devices inserted in circular cross-section conduits running full - Part 1: General principles and requirements. Geneva, Switzerland: ISO, 2003.

12. ISO 5167-4:2003. Measurement of fluid flow by means of pressure differential devices inserted in circular cross-section conduits running full - Part 4: Venturi tubes. Geneva, Switzerland: ISO, 2003.

13. Mottram RC. Damping criteria for pulsating gas flow measurement. Flow Meas Instrum 1989; 1: 15-23.

14. Reader-Harris M. Orifice plates and venturi tubes. Experimental Fluid Mechanics. Cham: Springer, 2015.

15. Schäfer C, Sandor I and Baar R. Improvement of the performance prediction of a small centrifugal compressor for automotive applications using computational fluid dynamics. In: Proceedings of the 12th international symposium on experimental and computational aerothermodynamics of internal flows, Lerici, Italy, 13-16 July 2015, paper no. ISAIF12-004.

16. Pucher $\mathrm{H}$ and Mai H. TC mapping - Parameterstudie zur Turbolader-Kennfeldvermessung. Final Report, VFI, 2010.

\section{Appendix}

Notation

$\begin{array}{ll}c_{D} & \text { flow rate coefficient }(-) \\ D_{1} & \text { flowmeter inlet diameter }(\mathrm{mm}) \\ D_{2} & \text { flowmeter throat diameter }(\mathrm{mm}) \\ D_{3} & \text { flowmeter outlet diameter }(\mathrm{mm}) \\ L & \text { length of airpath }(\mathrm{mm}) \\ \dot{m} & \text { mass flow }(\mathrm{kg} / \mathrm{s}) \\ p & \text { static pressure }(\mathrm{Pa}) \\ R & \text { gas constant }(\mathrm{J} / \mathrm{kg} \cdot \mathrm{K}) \\ t_{X F} & \text { crossfade duration }(\mathrm{s}) \\ T & \text { temperature }(\mathrm{K}) \\ x & \text { crossfade multiplier }(-) \\ \beta & \text { diameter ratio }(-) \\ \delta & \text { opening angle }\left({ }^{\circ}\right) \\ \Delta p & \text { differential pressure }(\mathrm{Pa}) \\ \varepsilon & \text { expansion number }(-) \\ \mu & \text { correction factor }(-)\end{array}$

\section{Subscript \\ A side facing compressor \\ $B \quad$ side facing flow volume \\ id idealized \\ ref reference measurement}

